\title{
Determinação das curvas-chave de descargas de sedimentos em suspensão na bacia hidrográfica do Rio Piancó-Piranhas-Açu
}

\author{
Determination of key curves of discharges of suspended \\ sediment in the hydrographic basin of Piancó-Piranhas-Açu River
}

Jose Wagner Alves Garrido', Thâmara Martins Ismael de Sousa², Luara Lourenço Ismael ${ }^{3}$,
Diêgo Lima Crispim ${ }^{4}$, Paloma Mara de Lima Ferreira ${ }^{5}$, Manoel Móises Ferreira de Queiroz

口-

\begin{abstract}
RESUMO
O estudo da descarga de sólidos de uma determinada bacia hidrográfica é importante para que se possam tomar decisões corretas quanto ao planejamento de gestão dos recursos hídricos. O objetivo do presente trabalho foi determinar curvas-chave que representam cargas de sedimentos em suspensão no Rio Piranhas. O estudo foi realizado na sub-bacia hidrográfica do Rio Piranhas. Foram realizadas 15 campanhas de medições hidrossedimentométricas, no período de novembro de 2012 a maio de 2013, envolvendo as medições de concentração de sedimentos em suspensão e de descargas líquida e sólida. Foram plotadas curvas-chave de sedimentos em suspensão para os períodos seco e chuvoso, as quais apresentaram bons coeficientes de determinação.
\end{abstract}

Palavras-chave: sedimentos; vazão; curva-chave; Rio Piranhas.

\begin{abstract}
The study of solid discharge of a hydrographic basin is important for making correct decisions regarding planning the management of water resources. The objective of the present study was to determine key planning curves that represented loads of suspended sediment in the River Piranhas. The study was carried out in the hydrographic sub-basin of Piranhas River. A total of 15 campaigns of hydrosedimentometric measurements was performed, in the period from November 2012 to May 2013, including the measurements of suspended sediment concentration and liquid and solid discharges. Key curves were plotted for sediment in suspension, for the dry and rainy periods, which presented good coefficients of determination.
\end{abstract}

Keywords: sediments; flow; keys curve; Piranhas River.

\section{INTRODUÇÃO}

Os processos sedimentológicos que ocorrem na natureza têm sido acelerados e intensificados pela atividade humana, destacando-se o uso inadequado do solo, o desmatamento, a urbanização, a atividade agropecuária e a alteração dos cursos dos rios (BRITO et al., 2009).

O sedimento presente no curso d'água é originado da produção de sedimentos da bacia hidrográfica. Em ocasiões de chuva, o escoamento superficial decorrente transporta muitas partículas para o rio onde esse sedimento se move em suspensão ou no leito, rolando, deslizando ou em saltos. Em suspensão no meio líquido encontram-se, em maior quantidade, partículas finas, como argila e silte; de 70 a 95\% da carga sólida total de sedimento é composta dessas partículas, sendo estas contempladas por sua fácil determinação por apresentarem-se em maior quantidade (CARVALHO, 2008).

De acordo com Oliveira e Cabral (2011), valores de descarga sólida em suspensão, concentração de sedimentos em suspensão e outros parâmetros derivados dos estudos hidrossedimentológicos (por exemplo, produção de sedimentos, uso do solo e degradação de leito de rios) são de aplicação em muitas áreas de estudos, como:

'Doutorando em Engenharia Química pela Universidade Federal do Rio Grande do Norte (UFRN) - Natal (RN), Brasil.

2Doutoranda em Engenharia Civil e Ambiental pela Universidade Federal da Paraíba (UFPB) - João Pessoa (PB), Brasil.

${ }^{3}$ Doutoranda em Recursos Naturais pela Universidade Federal de Campina Grande (UFCG), Campina Grande (PB), Brasil.

${ }^{4}$ Doutorando em Engenharia Civil pela Universidade do Pará - Belém (PA), Brasil.

${ }^{5}$ Doutoranda em Engenharia Civil e Ambiental pela UFPB - João Pessoa (PB), Brasil.

${ }^{6}$ Professor adjunto na UFCG - Pombal (PB), Brasil.

Endereço para correspondência: Jose Wagner Alves Garrido - Universidade Federal do Rio Grande do Norte - Programa de Pós-graduação em Engenharia Química, Núcleo Tecnológico - Avenida Salgado Filho, Campus Universitário - 59078-970 - Lagoa Nova (RN), Brasil - E-mail: josewagnerag@gmail.com.br

Recebido em: 03/08/2014 - Aceito em: 21/12/2016 - Reg. ABES: 138711 
- o conhecimento da descarga sólida em suspensão e da concentração é muito importante nos estudos de tratamento d'água para abastecimento e em estudos ambientais ligados à fauna e à flora fluvial;

- a concentração e a descarga em suspensão são importantes em estudos de irrigação;

- descarga de sedimentos em suspensão, concentração de sedimentos em suspensão e parâmetros derivados de estudos são importantes no diagnóstico sedimentológico de uma bacia.

O acompanhamento dos fluxos de sedimentos ocorridos em um dado local da bacia permite o diagnóstico de eventuais impactos em sua área de drenagem ao longo do tempo, podendo tornar-se importante indicador ambiental. Tradicionalmente, a descarga sólida é determinada através da chamada curva-chave de sedimentos, que relaciona descarga sólida em suspensão e vazão líquida (CARVALHO, 1994).

O Rio Piancó-Piranhas-Açu, de domínio federal, nasce no município de Santa Inez, no estado da Paraíba, e segue seu curso natural pelo estado do Rio Grande do Norte, desaguando no Oceano Atlântico, na Costa Potiguar. Como a maioria absoluta dos rios do semiárido nordestino, à exceção do Rio São Francisco e do Parnaíba, é um rio intermitente em condições naturais. Os regimes hidrológicos desses rios sofrem fortes influências das condições climáticas do semiárido. Notadamente, no curto período chuvoso, ocorrido anualmente, onde as variações de vazões, da qualidade da água e da quantidade de sedimentos são decorrentes dos volumes de água superficiais, associadas às formas de uso do solo, às características fisiográficas das bacias e ao tipo de cobertura vegetal predominante da caatinga (CBHRPPA, 2013).

$\mathrm{O}$ aporte de sedimentos nessas bacias fica condicionado ao período chuvoso, com características bem peculiares às condições do semiárido. No início das chuvas, a caatinga encontra-se sem folhagem, deixando o solo exposto à ação de erosão da chuva.

$\mathrm{O}$ transporte de sedimentos é bem relatado em rios perenes em climas temperados ou úmidos, mas é menos estudado nos riachos de regiões áridas, apesar do conhecimento de sua alta eficiência de transporte (REID; LARONNE, 1995). Por essa razão, ainda são poucos os trabalhos publicados sobre transporte de sedimentos em rios em zonas semiáridas. Probst e Suchet (1992) destacam a falta de dados disponíveis para tais tipos de rios.

Pouco se sabe sobre o aporte da produção de sedimentos na bacia hidrográfica do Rio Piancó-Piranhas-Açu. Desse modo, o trabalho teve como objetivo determinar curvas-chave que representam cargas de sedimentos em suspensão no Rio Piranhas.

\section{METODOLOGIA}

No Quadro 1 está apresentado o fluxograma das atividades desenvolvidas durante a realização deste trabalho.

\section{Área de estudo}

O presente trabalho foi realizado na sub-bacia hidrográfica do Rio Piranhas (Figura 1), pertencente à bacia hidrográfica do Rio Piancó-Piranhas-Açu. A secção de controle onde foi realizado o monitoramento está localizada geograficamente pelas coordenadas 6 $433^{\prime} 43^{\prime \prime} \mathrm{S}$ e $37^{\circ} 47^{\prime} 40^{\prime \prime} \mathrm{W}$, próxima à ponte sobre o Rio Piranhas, na BR-230, a 5 km da cidade de Pombal, Paraíba.

\section{Medições na secção de controle}

Foram realizadas 15 campanhas de medições no período de novembro de 2012 a maio de 2013, correspondentes às medições de vazão e coletas de amostras de água com sedimentos em suspensão durante o período de estiagem (seco), com frequência mensal, e durante o período chuvoso, seguindo as ocorrências de chuvas que provocassem a alteração da vazão do rio.

Todos os equipamentos utilizados para medições e coletas pertencem ao Laboratório de Hidrologia do Centro de Ciências e Tecnologia Agroalimentar (CCTA) da Universidade Federal de Campina Grande (UFCG), Campus de Pombal, Paraíba.

\section{Determinação da descarga líquida}

Para a determinação da vazão líquida, foi utilizado um perfilador acústico de corrente doppler (acoustic profiler corrent doppler - ADCP) e um medidor acústico de vazão, modelo RiverSurveyor S5 da Sontek, com base no manual do equipamento (SONTEK, 2009).Também foram determinadas outras variáveis hidrossedimentológicas (profundidade média do rio, largura do topo da seção do rio, velocidade média da água e outras).

Foi colocado um cabo graduado na seção para prender a embarcação, onde o ADCP foi fixado na lateral desta e conectado a um notebook (software river Surveyor live) fornecido pelo fabricante.

Em seguida, foram realizadas, em cada campanha, no mínimo duas travessias da seção do rio, quando foram registrados, simultaneamente: perfil de fundo ou batimetria, perfis e direções de velocidades, trajetória descrita pelo barco e outros parâmetros. $\mathrm{O}$ próprio software encarrega-se de registrar e processar as informações colhidas e calcular a vazão total da seção.

Quadro 1 - Atividades desenvolvidas neste trabalho.

\begin{tabular}{|c|c|c|c|}
\hline Medições & \multicolumn{3}{|c|}{ > Determinação da descarga líquida } \\
\hline de controle & \multicolumn{3}{|c|}{$>$ Amostragem de sedimentos em suspensão } \\
\hline \multirow[b]{2}{*}{$\begin{array}{l}\text { Análises em } \\
\text { Laboratório }\end{array}$} & \multirow[b]{2}{*}{$\begin{array}{c}>\text { Análise de } \\
\text { sedimentos } \\
\text { em suspensão }\end{array}$} & \multicolumn{2}{|c|}{$\begin{array}{l}\text { • Determinação da concentração } \\
\text { de sedimentos em suspensão }\end{array}$} \\
\hline & & $\begin{array}{c}\text { • Determinação } \\
\text { da descarga } \\
\text { sólida em } \\
\text { suspensão }\end{array}$ & $\begin{array}{c}\text { • Determinação } \\
\text { da descarga } \\
\text { sólida total }\end{array}$ \\
\hline
\end{tabular}




\section{Amostragem de sedimentos em suspensão}

O processo de amostragem de sedimentos para a análise de concentração de sedimentos em suspensão foi baseado na metodologia de Carvalho (2008), usando a integração na vertical do perfil de igual incremento de largura.

Foram utilizados os amostradores DH-48 (medição a VAU - forma que é realizada por um operador que atravessa o curso de água a pé) com bico $1 / 4$ " e DH-49, fazendo uso do barco de alumínio da Aruak 500 Premium, com guincho fluviométrico modelo 207/10 da Hidromec e motor de poupa da Mercury.

O número de amostras obtidas variou de acordo com a época da campanha realizada. No período seco, o volume coletado foi geralmente $10 \mathrm{~L}$; no período chuvoso, cerca de $5 \mathrm{~L}$ para diferentes verticais.

\section{Análises em laboratório de sedimentos em suspensão}

A análise de sedimentos em suspensão foi constituída da determinação da concentração de sedimentos, bem como da descarga do material sólido em suspensão.
As amostras coletadas foram levadas ao Laboratório de Hidrologia do CCTA da UFCG, colocadas em repouso por um período mínimo de 96 horas, para a decantação dos sedimentos a fim de se obter a redução de volume, conforme Carvalho et al. (2000).

Para a determinação da concentração de sedimentos em suspensão foi empregado o método do tubo de retirada pela base. Os tubos foram montados no Laboratório de Hidrologia, constituindo-se de tubos de vidro graduados em divisões de $5 \mathrm{~mm}$, com 1,20 m de comprimento, $2,54 \mathrm{~cm}$ de diâmetro interno e $3,0 \mathrm{~cm}$ de diâmetro externo, conforme descrito por Carvalho (2008).

A concentração de sedimentos em suspensão $\left(\mathrm{C}_{\mathrm{ss}}\right.$, em mg.L $\left.\mathrm{L}^{-1}\right)$ foi obtida a partir da Equação 1, fazendo a relação entre massa de sedimentos ( $\mathrm{m}_{\mathrm{ss}}$, em $\mathrm{mg}$ ) (sedimento seco) e o volume de água com sedimento coletado $(\mathrm{V}, \mathrm{em} \mathrm{L})$.

$\mathrm{C}_{\mathrm{ss}}=\frac{\mathrm{m}_{\mathrm{ss}}}{\mathrm{V}}$

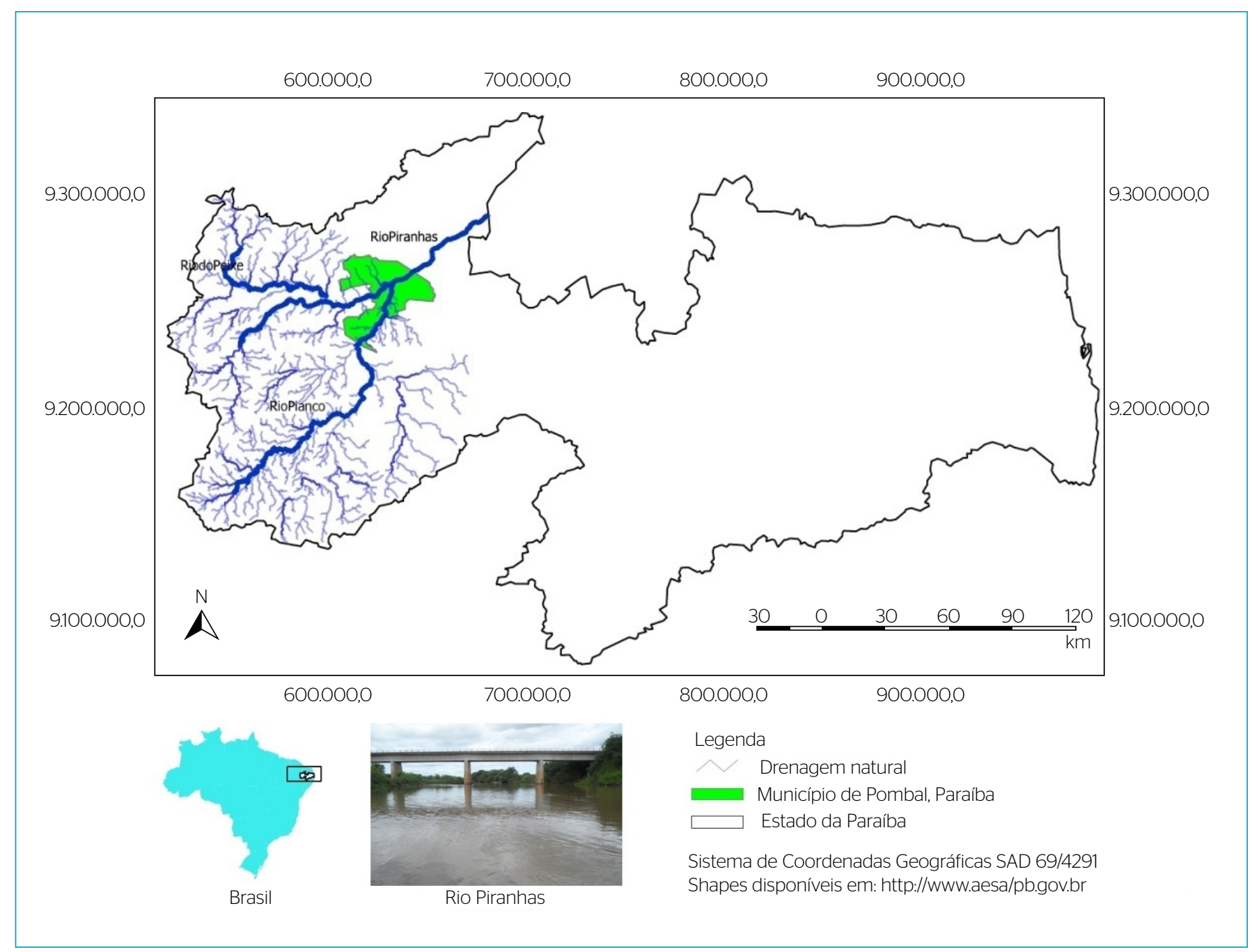

Figura 1 - Identificação da área de estudo, Rio Piranhas. 
As descargas sólidas em suspensão medida $\left(\mathrm{Q}_{\mathrm{ss}}\right.$, em t.dia $\left.{ }^{-1}\right)$, com amostragem de igual incremento de largura, foram calculadas a partir da Equação 2.

$\mathrm{Q}_{\mathrm{ss}}=0,0864 \cdot \mathrm{Q} \cdot \mathrm{C}_{\mathrm{ss}}$

sendo,

Q a descarga líquida $\left(\mathrm{m}^{3} \cdot \mathrm{s}^{-1}\right)$;

$\mathrm{C}_{\mathrm{ss}}$ a concentração de sedimento em suspensão $\left(\mathrm{mg} \cdot \mathrm{L}^{-1}\right)$.

No cálculo da descarga sólida total $\left(\mathrm{Q}_{\mathrm{st}}\right)$, a estimativa é efetuada com a concentração de sedimento menor do que a concentração real devido à limitação do equipamento, que não permite a coleta na zona não amostrada. Então, para essa correção pode ser aplicado o método modificado de Einstein (ou método de Colby, 1957), citado por Carvalho (2008).

Para o cálculo da $\mathrm{Q}_{\mathrm{st}}$, Colby (1957) desenvolveu pelo menos três métodos, baseados no método de Einstein e em várias medições no campo. Um deles, disponível em Carvalho (2008), é o que foi utilizado neste estudo, que usa basicamente três ábacos, dados de descarga líquida $\left(\mathrm{m}^{3} \cdot \mathrm{s}^{-1}\right)$, velocidade média $\left(\mathrm{m} . \mathrm{s}^{-1}\right)$, profundidade média do rio $(\mathrm{m})$, largura da seção do rio $(\mathrm{m})$ e concentração medida de sedimentos em suspensão (mg. $\left.\mathrm{L}^{-1}\right)$.

$\mathrm{A}_{\mathrm{st}}$ é calculada pela soma da $\mathrm{Q}_{\mathrm{ss}}$ com a descarga sólida não medida $\left(Q_{n m}\right)$, conforme a Equação 3 .

$\mathrm{Q}_{\mathrm{st}}=\mathrm{Q}_{\mathrm{ss}}+\mathrm{Q}_{\mathrm{nm}}$ em que,

$\mathrm{Q}_{\mathrm{st}}$ é a descarga sólida total (t.d $\left.\mathrm{d}^{-1}\right)$;

$\mathrm{Q}_{\mathrm{ss}}$ é a descarga sólida medida ( $\left.\mathrm{t} \cdot \mathrm{d}^{-1}\right)$;

$\mathrm{Q}_{\mathrm{nm}}$ é a descarga sólida não medida (t.d $\left.{ }^{-1}\right)$.

$\mathrm{A}_{\mathrm{nm}}$ é determinada a partir do produto da descarga sólida não medida aproximada pelo fator de correção e pela largura da seção, conforme a Equação 4.

$\mathrm{Q}_{\mathrm{nm}}=\mathrm{q}_{\mathrm{nm}} \cdot \mathrm{K} \cdot \mathrm{L}$

onde,

$\mathrm{Q}_{\mathrm{nm}}$ é a descarga sólida não medida (t.d $\left.{ }^{-1}\right)$;

$\mathrm{q}_{\mathrm{nm}}$ é a descarga sólida não medida aproximada $\left(\mathrm{t} \cdot \mathrm{d}^{-1} \cdot \mathrm{m}^{-1}\right)$;

K é o fator de correção (adimensional);

L é o comprimento (m).

\section{RESULTADOS E DISCUSSÃO}

A região semiárida é caracterizada por períodos sazonais distintos, um período de estiagem e outro período chuvoso. Diante disso, observa-se que as variáveis hidrossedimentológicas estudadas apresentaram diferentes relações para os dois períodos, conforme observado na Tabela 1, que apresenta os resultados das análises de sedimentos em suspensão e dos parâmetros hidrossedimentológicos registrados.

Tabela 1 - Dados das análises de sedimentos em suspensão do Rio Piranhas.

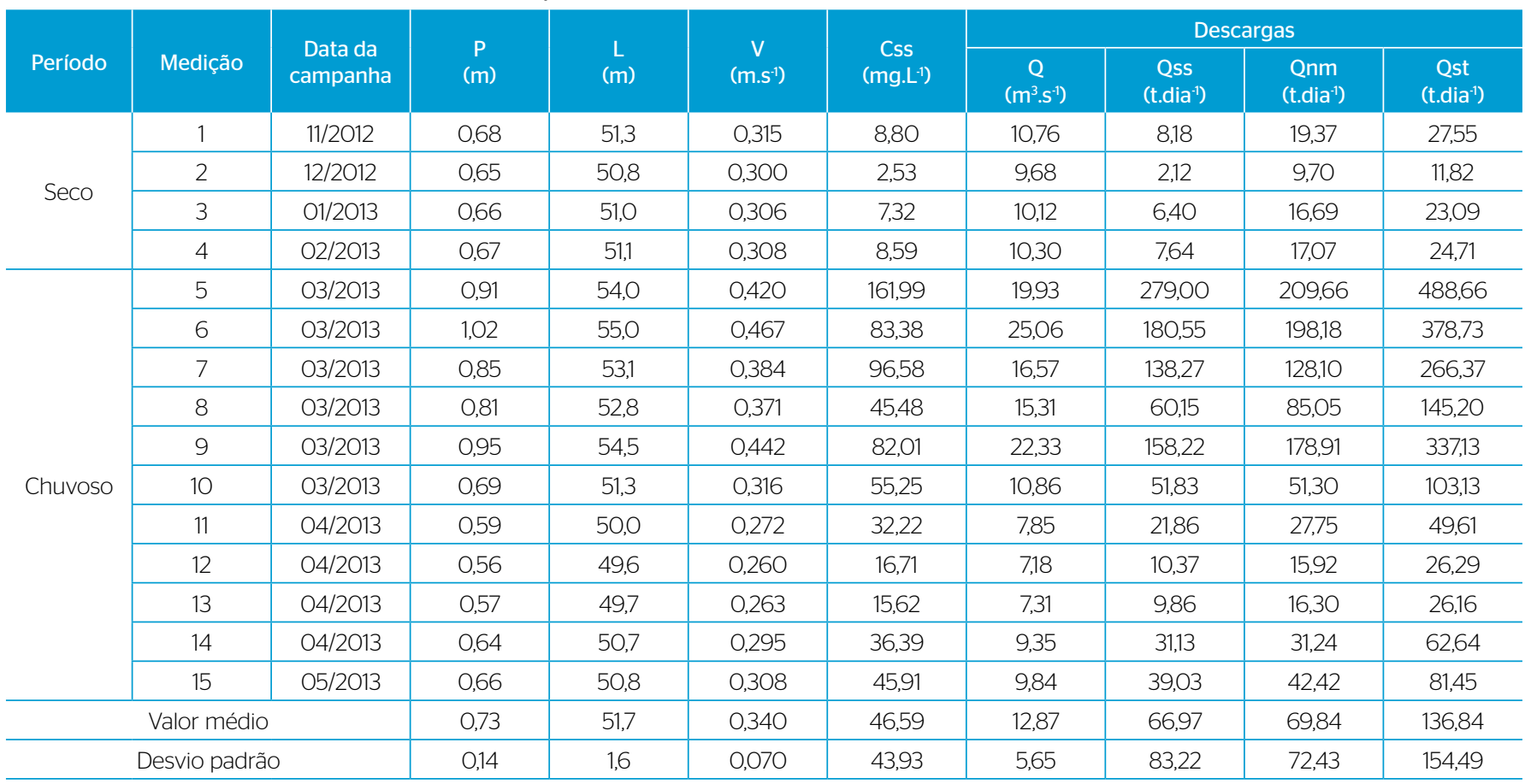

P: profundidade; L: largura do topo; V: velocidade média da água; Q: vazão; $C_{s s}$ : concentração de sedimentos em suspensão; $Q_{s:}$ : descarga sólida em suspensão medida;

$\mathrm{Q}_{\mathrm{nm}}$ : descarga sólida em suspensão não medida; $\mathrm{Q}_{\mathrm{st}}$ : descarga sólida total. 
Os valores das $\mathrm{C}_{\mathrm{ss}}$ variaram entre 2,53 e 161,99 mg.L $\mathrm{L}^{-1}$, tendo um valor médio de 46,59 mg. $\mathrm{L}^{-1}$ com desvio padrão de 43,93 mg. $\mathrm{L}^{-1}$ para uma vazão média de $12,87 \mathrm{~m}^{3} \cdot \mathrm{s}^{-1}$. O valor médio da $\mathrm{C}_{\mathrm{ss}}$ encontrado no Rio Piranhas difere dos valores encontrados em alguns outros rios com diferentes vazões, como mostram os estudos realizados por: Lima et al. (2004), no Rio Tocantins, com uma concentração de $8,84 \mathrm{mg} \cdot \mathrm{L}^{-1}$ para uma vazão média de $10.981,0 \mathrm{~m}^{3} \cdot \mathrm{s}^{-1}$; Lima (2001), no Rio São Francisco, com um valor médio de $27,38 \mathrm{mg} . \mathrm{L}^{-1}$ e vazão média de $2.119,5 \mathrm{~m}^{3} \cdot \mathrm{s}^{-1}$; Guyot, Filizola, Laraque (2005) que, trabalhando no Rio Amazonas, encontraram uma concentração de 149,33 mg.L-1 e vazão média de $149,33 \mathrm{~m}^{3} \cdot \mathrm{s}^{-1}$; Carvalho et al. (2005), no Rio Paraguai, que observaram uma concentração de $108,11 \mathrm{mg}$. $\mathrm{L}^{-1}$ e vazão média de $172.000,0 \mathrm{~m}^{3} . \mathrm{s}^{-1}$; e Souza (2011), no Rio Capibaribe, com uma concentração de 561,95 mg. $\mathrm{L}^{-1}$ e vazão média de $2,49 \mathrm{~m}^{3} \cdot \mathrm{s}^{-1}$.

Os dados apresentados na Tabela 1 , referentes aos meses de novembro de 2012 a fevereiro de 2013, correspondem ao período de estiagem (seco), que começa em setembro e se prolonga até fevereiro; os meses de março a maio compõem o período chuvoso, que se prolonga até agosto.

No período seco, ocorreu baixa produção de sedimentos, constatando-se que a menor $\mathrm{C}_{\mathrm{ss}}$ ocorreu na segunda medição, com um valor correspondendo a $2,53 \mathrm{mg} \cdot \mathrm{L}^{-1}$ para uma vazão de $9,68 \mathrm{~m}^{3} \cdot \mathrm{s}^{-1}$. Já no mês de março, quando foi registrada ocorrência de chuva significativa por estar no início da época chuvosa, registrou-se a maior concentração, que ocorreu na quinta medição, com um valor de 161,99 mg. $\mathrm{L}^{-1}$ para uma vazão de $19,93 \mathrm{~m}^{3} \cdot \mathrm{s}^{-1}$.

Essa variação de produção de sedimentos também foi avaliada por Souza (2011) no estudo referente à produção de sedimentos da bacia hidrográfica do Rio Capibaribe. Para o período chuvoso e não chuvoso do ano de 2010 constata que a maior $\mathrm{C}_{\mathrm{ss}}$ ocorreu no período chuvoso com um valor de $1.071,55 \mathrm{mg} . \mathrm{L}^{-1}$ para uma vazão de $5,31 \mathrm{~m}^{3} \cdot \mathrm{s}^{-1}$, e a menor, no período não chuvoso, com um valor de $363,10 \mathrm{mg} \cdot \mathrm{L}^{-1}$ para uma vazão de $0,29 \mathrm{~m}^{3} \cdot \mathrm{s}^{-1}$.

De acordo com Carvalho (2008), que analisou o comportamento das concentrações de sedimentos, de 70 a $90 \%$ do material transportado nos cursos d'água está diretamente relacionado à quantidade de precipitações e ocorrem principalmente durante o período de fortes chuvas. Este caso também foi observado no Rio Piranhas, de acordo com a Tabela 1, na primeira medição do mês de março, quando se obteve maior registro de $\mathrm{C}_{\mathrm{ss}}$ neste estudo, devido à ocorrência das primeiras precipitações no dia anterior da amostragem, em toda a bacia hidrográfica do Rio Piranhas.

No período de início das chuvas, na região de estudo, a vegetação encontrava-se sem folhagem, deixando o solo exposto à ação do processo erosivo e propiciando a lavagem de toda a bacia hidrográfica pelo escoamento superficial, carreando todo o material sólido desprendido para o exutório, promovendo o registro de maior $\mathrm{C}_{\mathrm{ss}}$ no período estudado.

Seguindo o que descreve Carvalho (2008), a plotagem de uma grande quantidade de dados hidrológicos de uma determinada seção pode apresentar curvas distintas, uma do período de estiagem e outra do período chuvoso. Sendo assim, foram plotados os gráficos que representam curvas-chave de sedimentos em suspensão do Rio Piranhas para esses dois períodos, apresentadas nas Figuras 2 e 3, respectivamente. Essas curvas mostram a correlação existente entre a concentração ou descarga sólida em função da vazão ou descarga líquida.

Com os ajustes das curvas-chave das descargas sólida em suspensão e total do Rio Piranhas, para o período de estiagem (Figura 2),

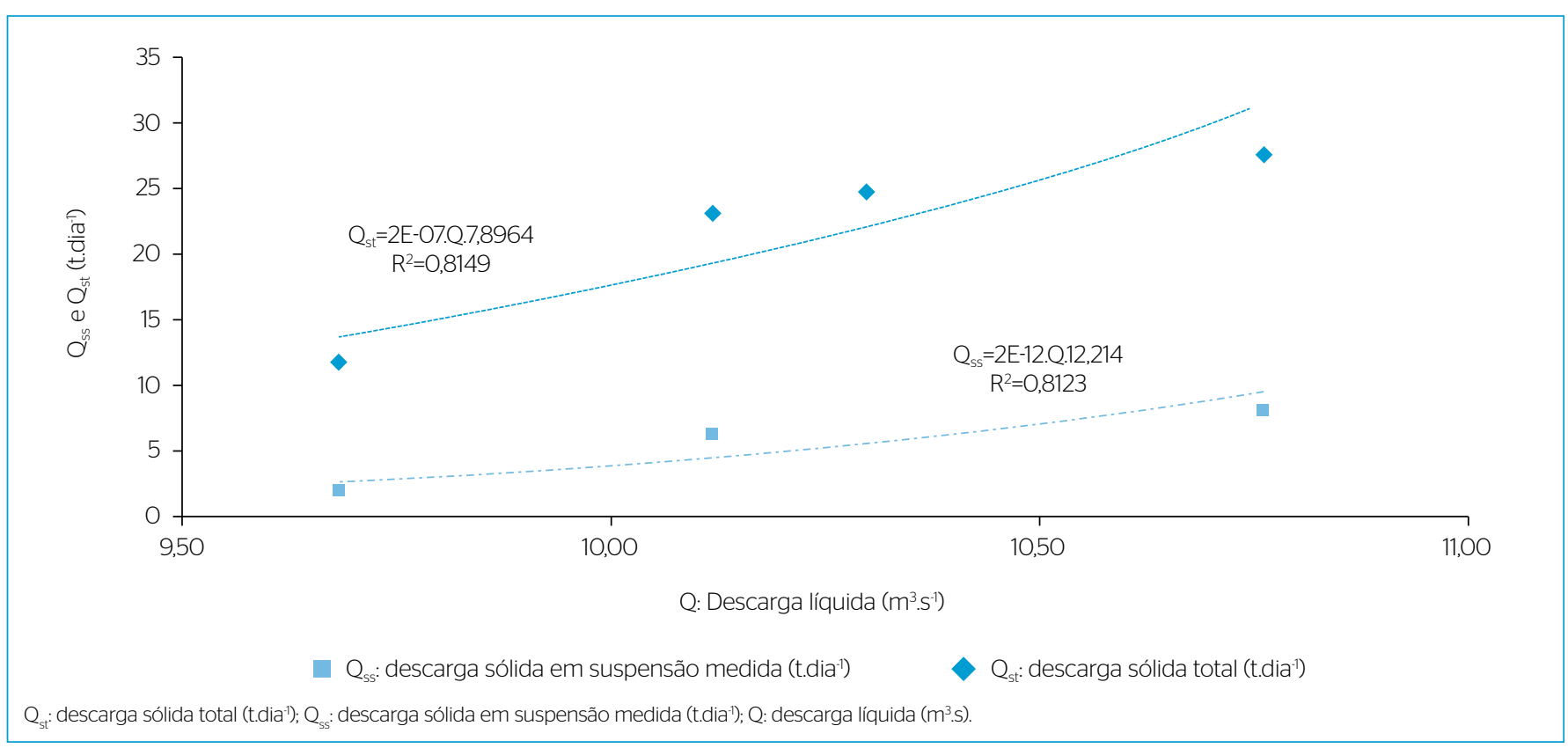

Figura 2 - Curvas-chave de sedimentos em suspensão do Rio Piranhas, para o período de estiagem (seco). 
pode-se observar que, mesmo utilizando poucos dados de observação, ocorreram bons ajustes das curvas. Os respectivos coeficientes de determinação $\mathrm{R}^{2}$, com valores de 0,8123 e 0,8149, respectivamente.

Os ajustes das curvas-chave das descargas sólida em suspensão e total do Rio Piranhas para o período chuvoso (Figura 3) também apresentaram boa correlação, como mostram os respectivos coeficientes de determinação 0,903 e 0,937.

Segundo Gomes et al. (2009), para se ter uma análise mais consistente das curvas é necessário um maior número de dados por se tratar de séries hidrológicas, principalmente na região do semiárido devido à desuniformidade das chuvas.

Os valores de descarga sólida em suspensão para o total de campanhas realizadas no Rio Piranhas variaram entre 180,55 e 2,12 t.dia ${ }^{-1}$ para as respectivas variações de descargas líquidas de 25,06 e 9,68 $\mathrm{m}^{3} \cdot \mathrm{s}^{-1}$.

Valores de descarga sólida em suspensão com magnitude bem superiores foram encontrados por Cunha Filho et al. (2010), em estudo de produção de sedimentos em suspensão da bacia hidrográfica do Riacho Exu, no semiárido Pernambucano, o qual teve variações de 13.539,39 a 1,03 t.dia ${ }^{-1}$; Melo et al. (2008), na pesquisa realizada na sub-bacia hidrográfica do Rio Jacu, em Pernambuco, obtiveram uma variação de 0,094 a 9,018 t.dia-1 com as respectivas variações de vazões de 0,00708 e $0,3446 \mathrm{~m}^{3} \cdot \mathrm{s}^{-1}$.

De acordo com Carvalho (2008), na natureza, para cada vazão num determinado momento existe um valor distinto de descarga sólida, concluindo que a curva-chave de sedimentos não pode substituir os valores reais confiáveis.

Chow, Maidment e Mays (1998) ressaltam que as curvas-chave precisam ser checadas periodicamente, devido às diferenças que podem ocorrer em relação a alguns fatores como a deposição de sedimentos, que para uma mesma cota pode fornecer vazões diferentes. Souza (2011) também ressalta que se deve ficar atento ao fato de a curva-chave estar intimamente ligada às características hidráulicas da seção de controle, pois qualquer mudança dessas características pode acarretar uma variação da expressão matemática.

\section{CONCLUSÕES}

A concentração de material transportado no Rio Piranhas está diretamente relacionada com a quantidade de precipitações; ou seja, as maiores $\mathrm{C}_{\mathrm{ss}}$ foram medidas no período chuvoso, sendo o maior valor (161,99 mg. $\left.\mathrm{L}^{-1}\right)$ registrado na primeira medição do mês de março, início da época chuvosa.

Tanto no período de estiagem quanto no período chuvoso, as curvas-chave de descarga sólida em suspensão e total apresentaram bons coeficientes de determinação, sendo, respectivamente, 0,8123 e 0,8149 no primeiro período e 0,9030 e 0,9370 no segundo.

As principais medidas preventivas que deveriam ser implantadas nas margens desse rio são a manutenção e a reconstituição das matas ciliares, conforme determina a Lei Florestal Brasileira. A implantação de matas ciliares é a ação preventiva mais importante para contenção de sedimentos, pois elas "filtram" a água proveniente das chuvas que escoam para o reservatório, evitando problemas de assoreamento.

Espera-se, com este estudo, ter contribuído para a compreensão e para a difusão do conhecimento do aporte e transporte de sedimentos do Rio Piranhas, servindo como um ponto de partida para outros trabalhos dessa natureza.

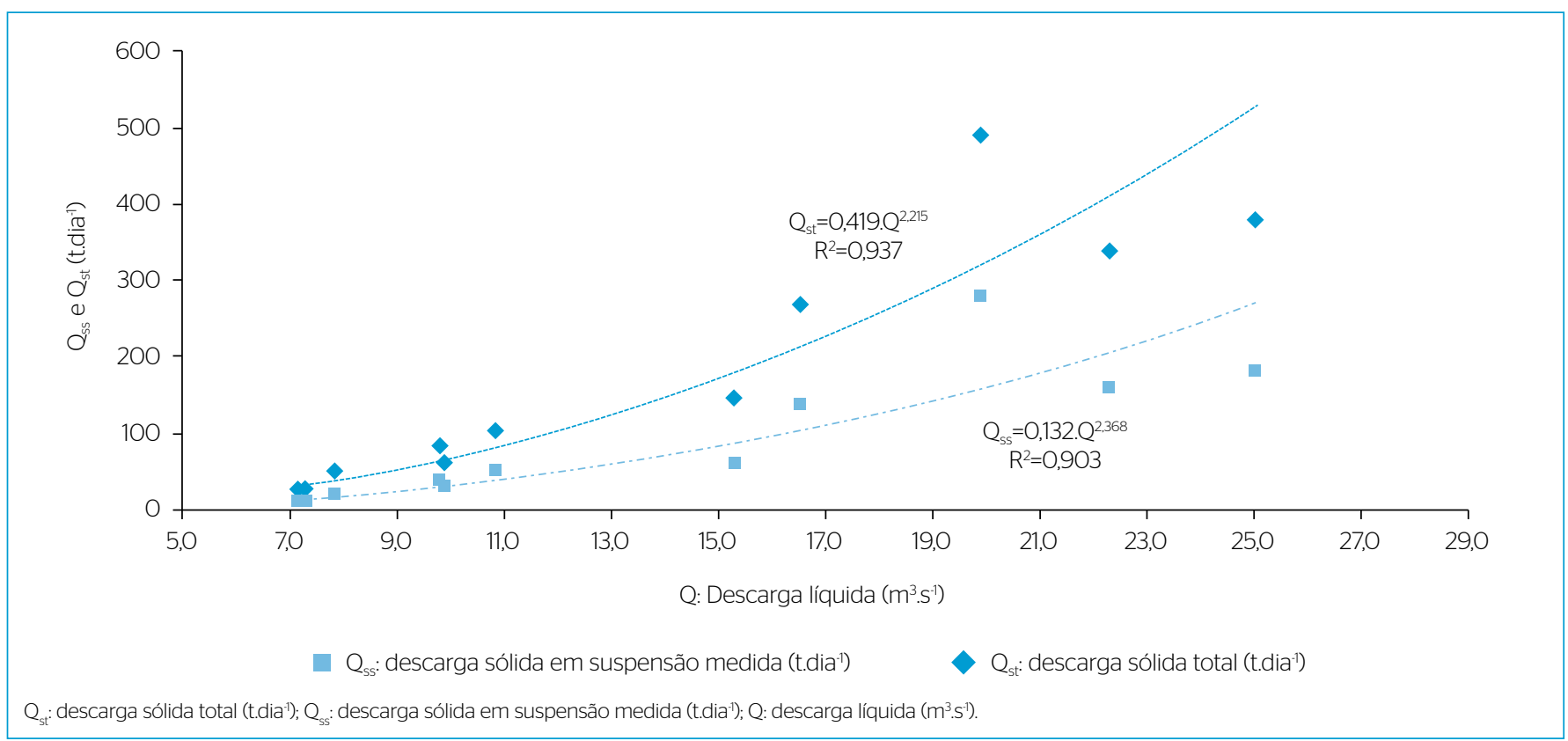

Figura 3 - Curvas-chave de sedimentos em suspensão do Rio Piranhas, para o período chuvoso. 


\section{REFERÊNCIAS}

BRITO, R. N. R.; ASP, N. E.; BEASLEY, C. R.; SANTOS, H. S. S. (2009) Características sedimentares fluviais associadas ao grau de preservação da mata ciliar-rio Urumajó, nordeste paraense. Revista Acta Amazônica, v. 39, n. 1, p. 173-180.

CARVALHO, N.O. (1994) Hidrossedimentometria prática. Rio de Janeiro: CPRM - Eletrobras. 372 p.

(2008) Hidrossedimentologia prática. 2. ed. Rio de Janeiro: Interciência.

CARVALHO, N.O.; IDE, C.N.; VAL, L.A.A.; RONDON, M.A.C.; BARBEDO, A.G.A.; CYBIS, L.F.A. (2005) Riscos devido à degradação e agradação de solos na bacia do alto Paraguai. In: SIMPÓSIO BRASILEIRO DE RECURSOS HIIDRICOS, 16., 2005, João Pessoa. Anais... João Pessoa: ABRH. CD-ROM.

CARVALHO, N.O; FILIZOLA JÚNIOR, N.P.; SANTOS, P.M.C.; LIMA, J.E.F.W. (2000) Guia de Práticas Sedimentométricas. Brasília: ANEEL. 154 p.

COMITEE DA BACIA HIDROGRÁFICA DO RIO PIANCÓ PIRANHAS ACÚ (CBHRPPA). A bacia. Disponível em: <http://www.cbhpiancopiranhasacu.org.br/site/a-bacia/s. Acesso em: 17 jun. 2013

CHOW, V.T.; MAIDMENT, D.R.; MAYS, L.M. (1998) McGraw-Hill series in water resources and environmental engineering. United States: Applied Hydrology; McGraw-Hill. 565 p.

CUNHA FILHO, M.; CANTALICE, J.R.B.; STOSIC, B.; ARAÚJO, A.M.; PISCOYA, V.C:; ALVES, P.S. (2010) Produção de sedimentos em suspensão da bacia hidrográfica do riacho exu no semiárido pernambucano. In: ENCONTRO NACIONAL DE ENGENHARIA DE SEDIMENTOS, 9., 2010, Brasília. Anais... Brasília.

GOMES, R.J.; JUNIOR, V.P.S.; MONTENEGRO, A.A.A.; SILVA, T.P.N. (2009) Avaliação da descarga líquida e sólida em seção natural da bacia do alto Ipanema-PE. In: JORNADA DE ENSINO, PESQUISA E EXTENSÃO, 9., 2009, Recife. Anais... Recife: UFRPE. CD-ROM.
GUYOT, J.L.; FILIZOLA, N.P.; LARAQUE, A. (2005) Régime et bilan du flux sédimentaire de l' Amazone à Óbidos (Pará, Brésil) de 1995 à 2003. In: SEDIMENT BUDGETS, 1., 2005, Foz do Iguaçu. Publication of International Association of Hydrological Sciences... v. 291. p. 347-354.

LIMA, J.E.F.W. (2001) Diagnóstico do fluxo de sedimentos em suspensão na bacia do rio São Francisco. Brasília: Embrapa Cerrados; ANEEL-ANA.

LIMA, J.E.F.W:; SANTOS, P.M.C:; CARVALHO, N.O.; SILVA, E.M. (2004) Diagnóstico do fluxo de sedimentos em suspensão na Bacia Araguaia Tocantins. Planaltina: Embrapa Cerrados; Brasília: ANEEL/ANA.

MELO, R.O.; CANTALICE, J.R.B.; ARAÚJO, A.M.; CUNHA FILHO, M. (2008) Produção de sedimento suspenso de uma típica bacia hidrográfica semiárida. In: ENCONTRO NACIONAL DE ENGENHARIA DE SEDIMENTOS, 8., 2008, Campo Grande. Anais... Campo Grande.

PROBST, J.L.; SUCHET, P.A. (1992) Fluvial suspended sediment transport and mechanical erosion in the Maghreb (North Africa). Journal of Hydrology, v. 37, n. 6, p. 621-637.

OLIVEIRA, S.F.; CABRAL, J.B.P. (2011) Análise da Concentração de sedimentos em suspensão e da descarga sólida da Bacia do rio Doce em Jataí GO. In: SIMPÓSIO BRASILEIRO DE RECURSOS HIDRÍCOS, 19., 2011, Maceió. Anais... Maceió-AL: ABRH. v. 1.

REID, I.; LARONNE, J.B. (1995) Bed load sediment transport in an ephemeral stream and a comparison with seasonal and perennial counterparts. Water Resource Research, v. 31, n. 3, p. 773-781.

SONTEK. (2009) Manual do Sistema RiverSurveyor S5/M9 versão de software 1.0. 9940 summers ridge road. San Diego, CA, EUA. 131 p.

SOUZA, W.L. da S. (2011) Produção de sedimentos da bacia hidrográfica do rio Capibaribe para zona costeira da região metropolitana do Recife. 136f. Dissertação (Mestrado) - Programa de Pós-Graduação em Ciência do Solo, Universidade Federal Rural de Pernambuco, Recife. 